\title{
A Análise de Grupamentos: uma Contribuição à Padronização do Projeto*
}

\section{Ricardo Manfredi Naveiro}

Professor adjunto do Programa de Engenharia de Produção da COPPE e-do Departamento de Engenharia Mecânica da Escola de Engenharia da UFRJ.

Consultor industrial.

Editor da Revista PRODUÇĀO.

Caixa Postal 68507 - Cidade Universitária-21945-Rio de Janeiro/RJ

\section{Ivan da Costa Pereira Filho}

Engenheiro mecânico

Mestrando do Departamento de Engenharia Mecânica da UFSC.

Palavras chave: projeto do produto, tecnologia de grupo, análise de grupamentos.

Key words: product design, group technology, cluster analysis.

\begin{abstract}
RESUMO
Este artigo apresenta um método para verificăção da semelhança entre duas peças, baseado nas técnicas de análise de grupamentos. O método é indicado para uso na etapa de projeto detalhado como auxílio à decisão na tarefa de especificação completa da geometria das peças. Os resultados indicam que o uso adequado deste método na etapa de projeto detalhado pode reduzir o universo de peças projetadas, aumentando o grau de padronizaçāo do produto.
\end{abstract}

\begin{abstract}
This paper presents a method for shape similarity verification based on cluster analysis techniques. The method was used for selection of components in the product detailed design stage. The results showed that the method can cont ribute for reducing company components spectrum, improving product standardization and company performance.
\end{abstract}

Rec. 07/91 Rev. 09/91 Apr.09/91

* Artigo apresentado na International Conference on Engineering Design - ICED91 realizada em Zürich em agosto de 1991.

PRODUÇÃO - Rio de Janeiro - Vol. 2 - N² - março 1992 - p.p. 157, 163 


\section{Introdução}

A atividade de projeto de produtos tem um importante papel na competitividade de uma empresa. Do ponto de vista do esforço técnico presente no desenvolvimento de produtos, existem requisitos estratégicosque influenciam o desempenho das empresas $(1,2)$ :

- capacidade para definir famílias de produtos que compartilhem custos de pesquisa e desenvolvimento;

- capacidade para reduzir os custos de fabricação, ainda na fase de projeto, utilizando os conceitos de "projeto simultâneo".

- capacidade para obter flexibilidade na produção.

A capacidade para reduzircustosdefabricação requer que o setor de projeto obtenha concepçōes de produto que compartilhem o maior número de recursos de manufatura disponíveis na empresa.

Oprocedimentode integrar-sea atividade de projeto com o planejamento do processo produtivo tem recebido a denominação de "projeto simultâneo".

Ele é principalmente utilizado no estágio de projeto detalhado, quando o produto já tem a sua concepção definida. Nesta época é que se completam as especificaçōes das diversas peças definindoas tolerâncias dimensionais, os acabamentos superficiais e identificando-se as ferramentase dispositivos necessários à fabricação. Nesta etapa é possível evitar a duplicação de projetos de componentes através do uso de sistemas de auxílio ao projeto que permitem ao projetista verificar se a peça/componente que ele precisa projetar está coerentecom os padrōes de produção da empresa (3). Levantamentos realizados em um conjunto de empresas americanas da área metalmecânica (4) indicaram que $19 \%$ das peças não necessitavam terem sido projetadas e que as $81 \%$ restantes apresentavam uma série de semelhanças entre si.

Este artigoapresenta um método deapoio à decisão de projeto baseado em análise de grupamentos capaz de atenderaos objetivos de padronização de componentes da empresa. O método pode ser aplicado tanto com a finalidade da redução de ferramental ou com a finalidade de redução de tempo de preparaçãode máquinas, por exemplo. Quem define o critério de padronização que se pretende alcançar é o conjunto de atributos utilizado para classificação.

\section{Métodos de classificação morfológica}

A aplicação do "projeto silmutâneo" na etapa de projetodetalhadoutiliza largamente conceitos desenvolvidos na tecnologia de grupo (5.6), na qual famílias de peças - que apresentam uma série de atributos comuns - são fabricadas da mesma forma, utilizando-se do mesmo conjunto de máquinas-ferramenta.

O diagrama apresentado na página seguinte mostra o fluxo de atividades do projeto num ambiente ondé utilizadoo projeto simultâneo. 


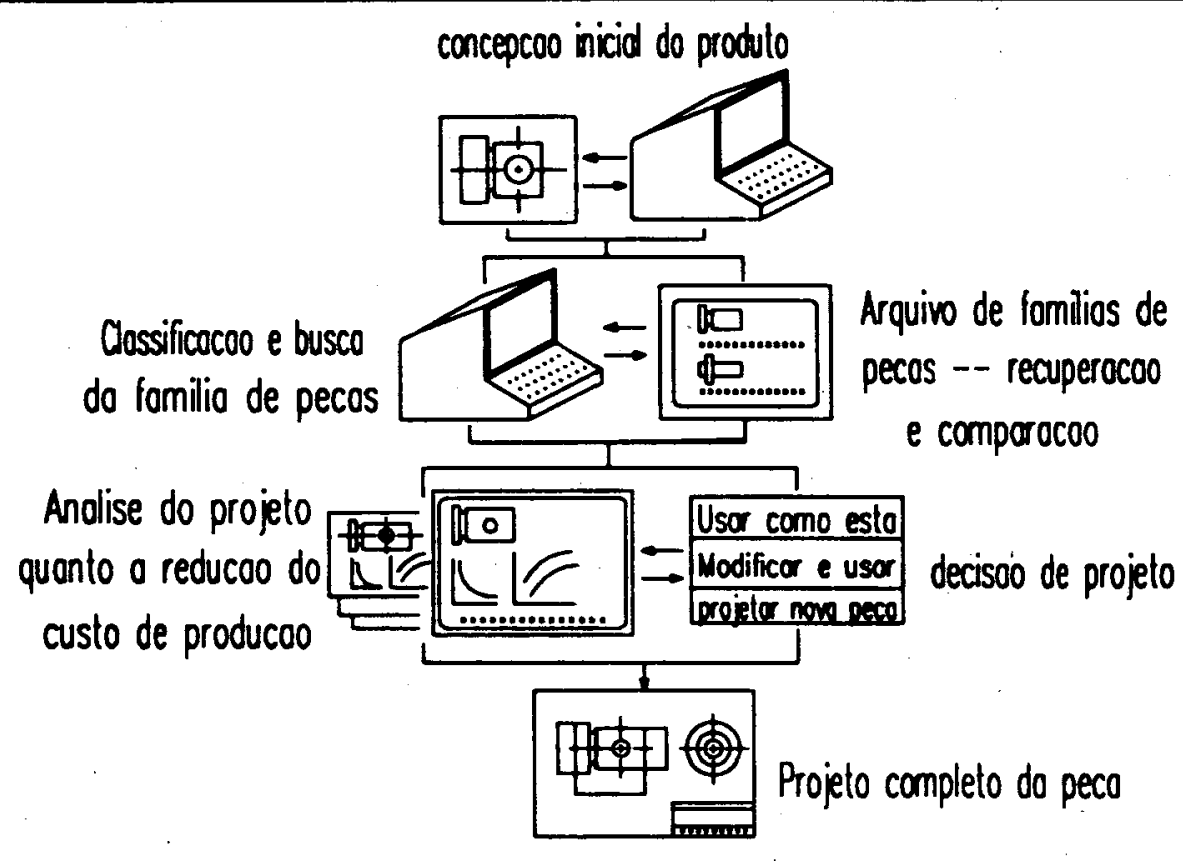

Diograma de fluxo para rocionalizacoo do projeto ( Ham,L. ,1987 )

O problema da formaçāo de famílias de peças já foi objeto de vários trabalhos $(7,8)$. Os métodos mais utilizados são: análise do fluxo de produção, classificaçāo e codificação e análise de grupamentos.

A análise de grupamentos tem o mesmo objetivo dos outros métodos: dividir um conjunto de peças quaisquer em grupos que apresentem determinadas características comuns. Inicialmente trata-se de um problema no qual nãose conhece a estrutura de grupos presente no universo pesquisado. Após a determinação da estrutura de famílias de peças, o problema passa a ser de classificação.

\section{Algorítmos para análise de grupamento}

Vários algorítmos de grupamento são citados nas publicações especializadas (Anderberd, 1973; Everitt, 1974; Villa, 1987), sendoa maior parte deles algorítmos heurís- 
ticos. O métodoheurístico utiliza uma medida de similaridade e um conjunto de regras de modo a atribuir elementos a uma classe, em oposição aos métodos de índice de desempenho que procuram maximizar ou minimizar um certoatributo de desempenho.

Algorítmos de grupamento podem ser ainda classificados como aglomerativos ou divisivos, de acordo com a séquência na qual as peças são grupadas. Um algorítmo aglomerativo parte de elementos isolados e grupa elementos próximos de acordo com um valor predefinido de baixo para cima. Inversamente, um algorítmodivisivo iniciase pelo conjunto de todos os elementos e o divide em grupos numa direção de cima para baixo, de acordo com critérios preestabelecidos.

Os elementos que estão sujeitos a grupamento podem ser de natureza variada: pessoas, opiniões, empresas, etc.. Neste trabalho os elementos são componentes de produtos industriais: peças dotadas de forma, textura, precisão dimensional, etc...

As peças apresentam um conjunto de características, atributos e propriedades denominado de conjunto de variáveis - que as descrevem total ou parcialmente. A escolha do conjunto de variáveis depende dos objetivos pelos quais está se fazendo análise de grupamentos. Nesta aplicação as variáveis selecionadas estão associadas ao objetivo de "padronização do projeto", através da redução da variedade de características diferentes de fabricação presentes nas peças.

O método quantifica o grau de similaridade existente entre os componentes de um produto através de uma medida de semelhança entre cada par de componentes. Foi desenvolvido um grande número de algorítmos degrupamentoe foram definidos vários tipos de coeficiente de similaridade.
A maioria dos trabalhosadota algorítmos hierárquicos aglomerativos e coeficiente de similaridade baseado na "distância" entre dois elementos. Dentre as diversas funçōes dedistância existentes, a "distância deHamming" é recomendada para aplicaçōes qualitativas, enquanto a "distância euclideana" deve ser utilizada para os casos de similaridade quantitativa(9).

O método aglomerativo reúne dois elementos, baseado na distância entre dois pares quaisquer ou, quando já existem gruposformados, na média das distâncias entre todos os pares formados a partir de um elemento de cada grupo. O procedimento aglomerativo é repetido até que todos os elementos estejam reunidos em um único grupo (10).

Cabe ao usuário definir o ponto no qual o processo deve ser interrompido, fornecendo o valor da "medida de semelhança" a ser utilizada. Neste trabalhodenominamoscoeficiente de similaridade o valor que expressa o nível de semelhança a ser alcançado.

\section{Sistema de auxílio à padronização do projeto}

O módulo "formação de famílias de peças" forma o conjunto inicial de famílias de peças - grupos de peças semelhantes em relação a um conjunto qualquer de atributos.

O procedimento deste módulo consiste em, inicialmente, associar a cada peça um vetor de características que representa a presença/ausência de determinadas características em cada uma das peças, resultando num vetor de variáveis binárias, onde o número 1 representa a presença da carácterística na peça e o número 0 o contrário. 


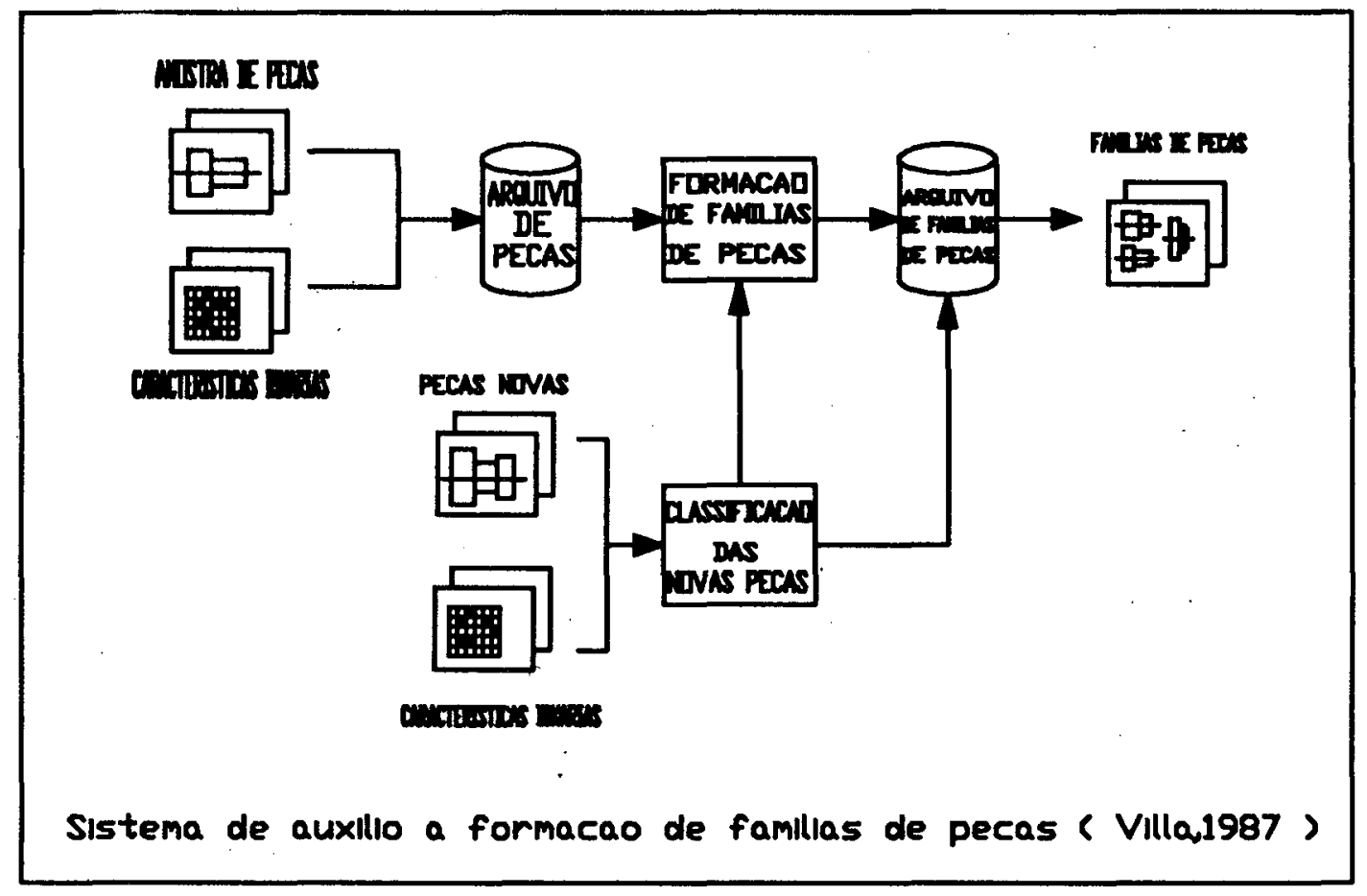

Este procedimentoé realizado pelo usuário interpretandoo esboçoeas especificaçōes das peças a serem grupadas.

A cada peça representada pelo vetor de características é atribuído um número de identificação único. Este número, seguido do vetor binário que descreve a peça representa uma linha do arquivo "amostra de peças".

Em seguida é processado o algorítmo hierarquizado aglomerativo para cada coeficiente de similaridade definido pelo usuário, gerando um conjunto de famílias de peças. A escolha da estrutura de grupos que melhor atende aos objetivos do trabalho é de responsabilidade do usuário.

O sistema é constituído de dois módulos principais:
O módulo "classificador de novas peças" classificadas peças que estão sendo projetadas verificando a pertinência da peça a uma das famílias existentes. Caso a peça não pertença a nenhuma das famílias existentes, osistema indica a família mais "próxima" da peça. Neste caso, as tarefas do projetista são: identificar quais os parâmetros que acarretam a falta de ajuste da peça à família, analisar as diversas possibilidades de modificação para promoveroajustee decidir pela sua implantação.

Para uma peça pertencer a uma determinada família ela precisa "distar" das demais um valor máximo limitado pelo coeficiente de similaridade. O módulo classificadorverifica essa condição, mas ele não tem meios de indicar ao projetista um conjunto de sugestões coerente para auxílio aoenquadramento da peça. 


\section{Exemplo de aplicação}

Verificou-se ofuncionamento domódulo "formação de famílias de peças" para um conjunto de 150 parafusos. Cada parafuso foi descrito através de 29 características relacionadas ao tipo de rosca, tipode cabeça, elementos geométricos específicos, etc. $\mathrm{O}$ desenho apresentado a seguir mostra o conjunto de oito características referentes à cabeça dos parafusos.

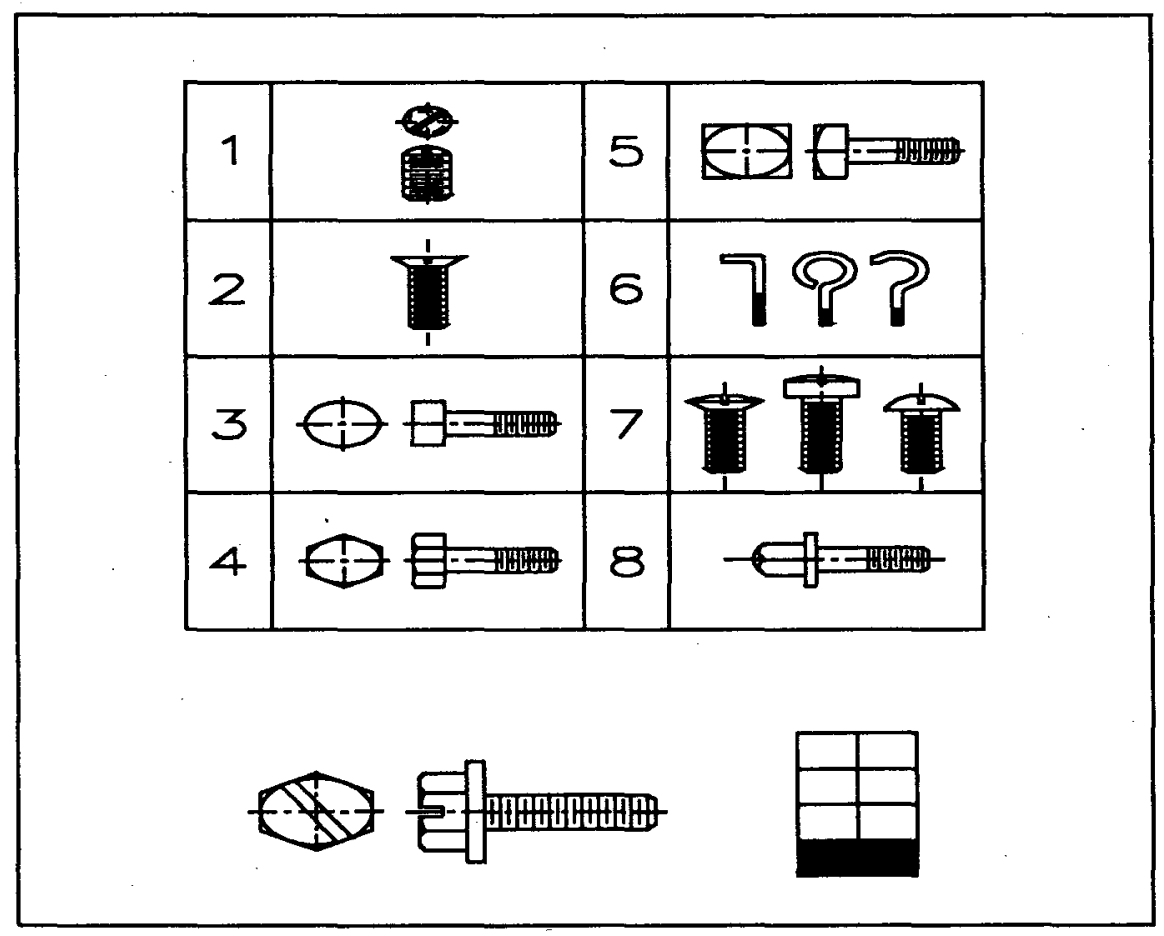

Formou-se um conjunto de 150 vetores de acordo com os atributos selecionados e procedeu-se à resolução do algorítmo para coeficientes de similaridade previamente escolhidos. O quadro a seguir dá uma idéia da relação entre o valor do coeficiente de similaridade e o número de famílias.

Coef. de similaridade $1,52,02,53,03,54,04,55,0102032,5$ No famílias

$\begin{array}{lllllllllll}30 & 25 & 21 & 19 & 15 & 14 & 12 & 10 & 3 & 1 & 1\end{array}$

O número 32,5 representa a maior distância entre dois parafusos quaisquer do conjunto.
Os principais resultados alcançados foram os seguintes:

- Os parafusos que foram repetidamente excluídos de grupos maiores, formando grupos unitários, são, conforme se pode observar, parafusos muito diferentes dos demais. O método foi, portanto, capaz de identificá-los e separá-los;

- Formaram-se umas poucas famílias com um número maior de peças que as demais. Este fato resulta da relativa homogeneidade da amostra e do pequeno número de características codificadas. O método foi, portanto, capaz de identificar as peças semelhantes e grupá-las. 


\section{Considerações finais}

O método de análise de grupamentos eo algorítmo implementado mostraram-se eficazes em relação aos objetivos propostos inicialmente.

Comparando-se com os métodos usuais de formação de famílias de peças, podemos identificaralgumas vantagens apresentadas pelo método de análise de grupamentos:

- possibilidade de formação de famílias de peças sem conhecimento prévio do número e composição das famílias.

- possibilidade de interação com bancos de dados de peças possibilitando uma via para a integração do projeto com o planejamento do processo produtivo.

- maior flexibilidade que o método de codificação, uma vezque pode seraplicado a vários objetivos diferenciados: padronização visando fabricação, padronização visando montagem, etc..., bastando mudarse o conjunto de características.

- possibilidade de atribuir-se pesos diferenciados àscaracterísticas, evidenciandose a im portância ou o poder descritivo de uma determinada característica em relação às demais.

O sistema de apoio à decisão no projeto apresentado neste trabalho constituiu-se na estrutura básica de um dispositivo técnico para auxiliar os projetistas a tomarem decisões voltadas aosobjetivos da empresa. Suas aplicações na red ução da geração desnecessária de novas peças representa um ganho efetivo de produtividade para as empresas.

\section{Bibliografia}

1. EEKELS J. - "On Strategy and Engineering Design" in Proceedings of the 1987 International Conference on Engineering Design, Asme, New York, 1987, Vol. 1, pp. 187-194
2. DIXON J. AND DUFFEY M. - "The Neglect of Engineering Design" in California Management Review, University of California Press, Berkeley, 1990, Vol. 32, no. 2, pp 9-23.

3. NAVEIRO R.M. - "Product Morphology and Production Automation", In Proceedings of the 1987 International Conference on Engineering Design, Asme, New York, 1987, Vol. 2, pp. 815-820.

4. HYDE W. - Improving productivity by classification, coding and data base standardization, MarcelDekker, New York, 1988.

5. ARN, E.A. - Group Technology-Springer Verlag, Berlin, 1975.

6. REMBOLD U., BLUME C. AND DILLMAN R. - Computer Integrated Manufacturing Technology and Systems Marcel Dekker, New York, 1985.

7. TOKNOKA, N., MAEDA T. AND SATO G. - "The Development of GT-CAD for definiton of views of machine parts" in Proceedings of the 1987 International Conference on Engineering Design, Asme, New York, 1987, Vol. 2, pp. 648-655

8. KNOSALA K. ANDPEDRYESW. - "Clyster Analysis and Fuzzy Classification In Design Methods" in Proceedings of the 1985 International Conference on Engineering Design, Heurista, Hamburg 1985, Vol. 1, 00. 66-72

9. VILLA E. - Computer Aided Group Technology Part Family Formation based on pettern recognition techniques-Ph.d pattern Thesis - The Pennsylvania State University - University Park - USA 1989

10. ANDERBERG M.R. - Cluster Analysis for Applications, Academic Press, New York, 1973.

(1) Em inglês é denominado concurret design e design for manufacturing

(2) O programa elaborado em Turbo Pascal para a resolução do algorítmo é baseado nos algorítmos exemplificados em Villa \& Anderberg. 